BULl. AUSTRAL. MATH. SOC.

VOL. 34 (1986) 87-92.

\title{
SEQUENCES AND BASES IN P-BANACH SPACES
}

\author{
M. A. ARiño
}

Necessary and sufficient conditions are given for an infinite dimensional subspace of a $p$-Banach space $X$ with basis to contain a basic sequence which can be extended to a basis of $X$.

In [1] it is proved that if $X$ is a Banach space with a basis and $\left(y_{n}\right)^{\infty} n=1$ is a regular sequence which converges to zero coordinatewise, then $\left(y_{n}\right)_{n=1}^{\infty}$ has a subsequence which can be extended to a basis of $X$ and so every infinite dimensional subspace $Y$ of $X$ contains a basic sequence which can be extended to a basis of $X$. Our purpose is to study these properties in $p$-Banach spaces.

If $X$ is a real linear space and $0<p \leqq 1$, a $p$-norm $\|\cdot\|$ on $X$ is a map from $X$ into $[0,+\infty)$ which satisfies the following conditions:

a) $\quad|x| \mid=0$ if and only if $x=0$.

b) $\|t x\|=|t|^{p}\|x\|$ if $x \in X$ and $t \in \mathbb{I R}$.

c) ||$x+y|| \leq\|x\|+\|y\|$ if $x, y \in X$.

Received 1 October 1985. The author would like to thank Professor Augustyn Ortyński for his suggestions.

Copyright Clearance Centre, Inc. Serial-fee: 0004-9727/86 $\$ A 2.00+0.00$. 
With the distance $d(x, y)=\| x-y||, X$ becomes a metric space and if $(X,\|\cdot\|)$ is complete, it is called $p$-Banach space (Banach space if $p=12$. The $p$-th root of a $p$-norm is a quasi-norm \|\|$\cdot\|\|$ and satisfies

a) $\|\mid x\|=0$ if and only if $x=0$.

b) $\|t x\||\|=|t|\||\|x\|$ if $x \in X$ and $t \in \mathbb{R}$

c) $\quad\|\mid x+y\| \| \leq C(\|x\|\|+\||\|\mid\|)$, where $C \geqq 1$ is a positive number which does not depend on $x$ and $y$.

In particular, the space $\ell_{p}(0<p<1)$ of all real sequences $\left(x_{n}\right)^{\infty} n=1$ such that

$$
\left\|\left(x_{n}\right)\right\|=\sum_{n=1}^{\infty}\left|x_{n}\right|^{p}<\infty
$$

is a p-Banach space.

Let $(X,|| \cdot||)$ be a $p$-Banach space with topological dual $X^{*}$, and suppose that $X^{*}$ separates the points of $X$ (this condition will be assumed throughout this paper). We can provide $X^{*}$ with the dual norm

$$
|| x^{*}||^{*}=\sup _{|| x|| \leq I}\left|x^{*}(x)\right|
$$

and $\left(X^{*},\|\cdot\|^{*}\right)$ becomes a Banach space. The inclusion map from $(X,\|\cdot\|)$ into $\left(X^{* *},\|\cdot\|^{* *}\right)$ is continuous, more precisely, $\left\|\left.x\right|^{* *} \leqq\right\| x \|^{1 / p}$ for every $x$.

If $(X,\|\cdot\|)$ is a $p$-Banach space, the convex hulls of the balls of $(X,\|\cdot\|)$ form a basis of zero neighbourhoods of a locally convex topology which is the finest locally convex topology on $X$ whose dual is $X^{*}$, that is, the Mackey topology of the dual pair $\left\langle X, X^{*}\right\rangle$. This is usually called the Mackey topology of $(X,\|\cdot\|)$, and can be defined by the norm induced by the bidual $\left(X^{* *},\|\cdot\|^{* *}\right.$ ) (see [3]).

$$
\text { If } Y \text { is a closed subspace of }(X,\|\cdot\|),(Y,\|\cdot\|) \text { is a } p-
$$
Banach space for which the bidual norm $\|\cdot\|_{Y}^{* *}$ defines the Mackey topology. In general, the Mackey topology of $Y$ is stronger than the topology induced by the Mackey topology of $X$. It is easy to see, using duality arguments, that both topologies coincide if and only if $Y$ has 
the Hahn Banach extension property (HBEP): every $y^{*} \epsilon Y^{*}$ is the restriction to $Y$ of some $x^{*} \in X^{*}$.

A sequence $\left(z_{n}\right)_{n=1}^{\infty}$ is called block basic sequence with respect to a basis $\left(x_{n}\right)_{n=1}^{\infty}$ if there exists a strictly increasing sequence of positive integers $\left(m_{n}\right)_{n=1}^{\infty}$ such that

$$
z_{n}=\sum_{i=m_{n-1}+1}^{m} a_{i} x_{i}
$$

where $\left(a_{n}\right)_{n=1}^{\infty}$ is a sequence of scalars. And a sequence $\left(y_{n}\right)^{\infty} n=1$ is called regular if $\inf _{n}\left\|y_{n}\right\|>0$. If $\left(x_{n}\right)_{n=1}^{\infty}$ is a bounded and regular basis of a $p$-Banach space, then $\inf _{n}\left\|x_{n}\right\|^{* *}>0$. (See [2, Proposition 3. 2.iii])

Let $\left(z_{n}\right)_{n=1}^{\infty}$ be a block basic sequence as $(*)$. If $\left(y_{n}\right)_{n=1}^{\infty}$ is a sequence in $X$ with $y_{m_{j}}=z_{j}$ and $y_{n} \in\left[x_{i}\right]_{i=m_{j-1}}^{m_{j}}$ whenever $m_{j-1}<n \leqq m_{j}$, then $\left(y_{n}\right)_{n=1}^{\infty}$ is called block extension of $\left(z_{n}\right)_{n=1}^{\infty}$. Morrow [5] has proved that a bounded and regular block basic sequence $\left(z_{n}\right)^{\infty} n=1$ in a $p$-Banach space $X$ has a block extension that is a basis of $X$ if and only if $\inf _{n}\left\|z_{n}\right\|^{* *}>0$.

The proof of the following lemma is similar to the one known for the Banach case (see $[4,1 . a .9]$ ) .

LEMMA 1. Let $\left(x_{n}\right)^{\infty}$. be a normalized basis of a p-Banach space $X$ with basis constant $K$. Let $\left(y_{n}\right)^{\infty} n=1$ be a sequence in $X$ with $\sum_{n=1}^{\infty}\left\|x_{n}-y_{n}\right\|<\frac{1}{2 K}$. Then $\left(y_{n}\right)_{n=1}^{\infty}$ is a basis of $x$ which is equivalent to $\left(x_{n}\right)_{n=1}^{\infty}$. 
THEOREM 2. Let $\left(x_{n}\right)^{\infty}{ }_{n=1}$ be a basis of a p-Banach space $X$ and $\left(Y_{n}\right)_{n=1}^{\infty}$ a normalized sequence in $X$ which converges to zero coordinatewise and such that $\inf _{n}|| y_{n} \|^{* *}=c>0$. Then $\left(y_{n}\right)_{n=1}^{\infty}$ has a subsequence which con be extended to a basis of $X$.

Proof. We can suppose that $\left(x_{n}\right)^{\infty}=1$ is normalized. Let $K$ be the basis constant of $\left(x_{n}\right)_{n=1}^{\infty}$. With an usual "gliding hump" method we can find a subsequence $\left(y_{p_{n}}\right)_{n=1}^{\infty}$ of $\left(y_{n}\right)^{\infty}{ }_{n=1}^{\infty}$ and a $\left(z_{n}\right)^{\infty}$ bl block basic sequence of $\left(x_{n}\right)_{n}$ as $(*)$ such that:

$$
|| y_{p_{n}}-z_{n}||<\frac{C}{K \cdot 2^{n+1}}
$$

since $\left\|y_{p_{n}}-z_{n}\right\|^{* *} \leqq\left\|y_{p_{n}}-z_{n}\right\|^{1 / p}<\frac{C}{4}$, we deduce that $\inf _{n}|| z_{n} \|^{* *}>0$ and therefore a $\left(u_{n}\right)_{n=1}^{\infty}$ block extension of $\left(z_{n}\right)_{n=1}^{\infty}$ exists, which is a basis of $X$. Let $\left(v_{n}\right)_{n=1}^{\infty}$ be the sequence defined by

$$
v_{n}= \begin{cases}u_{n} & \text { if } n \neq m_{k} \text { for every } k \\ y_{p_{k}} & \text { if } n=m_{k} .\end{cases}
$$

Since

$$
\sum_{n=1}^{\infty}\left\|v_{n}-u_{n}\right\|=\sum_{n=1}^{\infty}\left\|y_{p_{n}}-z_{n}\right\|<\frac{1}{2 K} .
$$

$\left(v_{n}\right)^{\infty}{ }_{n=1}$ is a basis of $x$ which is an extension of $\left(y_{p_{n}}\right)_{n=1}^{\infty}$ (lemma 1 is used).

THEOREM 3. Let $X$ be a p-Banach space with basis. Let $Y$ be an infinite dimensional subspace of $X$. The following are equivalent:

i) $Y$ contains a basic sequence which con be extended to a basis of $X$.

ii) The unit ball of $Y$ is not relatively compact in $\left(X^{* *},\|\cdot\| \|^{* *}\right)$. 
Proof. $i \Rightarrow$ ii) Let $\left(y_{n}\right)^{\infty} n=1$ be a basic sequence in $X$ such that a basis of $X\left(w_{n}\right)_{n=1}^{\infty}$ with $w_{p_{n}}=y_{n}$ for every $n \in \mathbb{I N}$ exists. We can suppose that $\left(y_{n}\right)_{n=1}^{\infty}$ is contained in the unit ball of $Y$. Let $\left(w_{n}^{*}\right)_{n=1}^{\infty} \subset X^{*}$ be the sequence such that $w_{n}^{*}\left(w_{m}\right)=\delta_{n, m}$ and we write $y_{n}^{*}=w_{p_{n}}^{*}$. If a subsequence $\left(y_{n_{j}}\right)_{j=1}^{\infty}$ of $\left(y_{n}\right)_{n=1}^{\infty}$ converges in $\left(X^{* *},|| \cdot||^{*}\right)$, then the sequence $\left(z_{j}\right)_{j=1}^{\infty}$ with $z_{j}=y_{n_{j}}-y_{n_{j+1}}$ converges to zero in $\left(X^{* *},\|\cdot\|^{* *}\right)$. But this is impossible because

$$
\left\|z_{j}\right\|^{* *} \geqq \frac{y_{n_{j}}^{*}}{\left\|y_{n_{j}}^{*}\right\|}\left(y_{n_{j}}-y_{n_{j+1}}\right)=\frac{1}{\left\|y_{n_{j}}^{*}\right\|} \geqq \frac{1}{K}
$$

where $K$ is the basis constant of $\left(w_{n}\right)_{n=1}^{\infty}$, and so the unit ball of $Y$ is not relatively compact in $\left(X^{* *},\|\cdot\|^{* *}\right)$.

ii $\Rightarrow i)$ If the unit ball of $Y$ is not relatively compact in $\left(X^{* *},\|\cdot\|^{* *}\right)$ then a bounded sequence $\left(z_{n}\right)^{\infty}{ }_{n=1}$ in $Y$ exists which does not have any subsequence converging in $\left(X^{* *},\|\cdot\|^{* *}\right)$. Replacing $\left(z_{n}\right)_{n=1}^{\infty}$ with a subsequence, if needed, we can suppose that $\left(z_{n}\right)_{n=1}^{\infty}$ is Cauchy-coordinatewise, and also assume that $0<\underset{n}{\inf }|| z_{n}-\left.z_{n+1}\right|^{* *}$. Then if $y_{n}=z_{n}-z_{n+1}$, applying theorem $1\left(y_{n}\right)^{\infty}$, has a subsequence which can be extended to a basis.

COROLLARY 4. If $Y$ has con infinite dimensional subspace $Z$ with HBEP then $Y$ contains a sequence which can be extended to a basis of $X$. Proof. If the unit ball of $(Y,\|\cdot\|)$ is relatively compact in $\left(X^{* *},\|\cdot\|^{* *}\right)$, the same is true for any subspace $Z$ of $Y$. If $Z$ has the HBEP, the Mackey topology of $(Z,\|\cdot\|)$ is defined by $\|\cdot\|^{* *}$ and $Z$ must be finite dimensional. 
THEOREM 5. Let $(X,\|\cdot\|)$ be a p-Banach space with basis, such that every infinite dimensional subspace $Y \subset X$ contains a basic sequence which can be extended to a basis of $X$. Then $(X,\|\cdot\|)$ must be locally convex.

Proof. If $(x,\|\cdot\|)$ is not locally convex, we can find a sequence $\left(x_{n}\right)^{\infty}$ in $X$ such that $\left\|x_{n}\right\|=1$ and $\left\|x_{n}\right\|^{* *}<\frac{1}{n}$. Using a "gliding hump" method we can assume that there exists a subsequence $\left(x_{n_{k}}\right)_{k=1}^{\infty}$ of $\left(x_{n}\right)_{n=1}^{\infty}$ which is a basic sequence. Let $y=\overline{s p}\left[x_{n_{k}}\right]_{k=1}^{\infty}$. since $\left\|x_{n_{k}}\right\|=1$ and $\left\|x_{n_{k}}\right\|^{* *}<\frac{1}{2^{n_{k}}}$ the inclusion map $i$ : $(Y,\|\cdot\|) \longrightarrow\left(X^{* *},\|\cdot\|^{* *}\right)$ is compact, and theorem 3 ensures us that $Y$ does not have any basic sequence which can be extended to a basis of $X$, contradicting the hypothesis.

\section{References}

[1] W. Dean, I. Singer, L. Sternbach, "On shrinking basic sequences in Banach spaces", Studia Math. 40, (1971), 23-33.

[2] N. J. Kalton, "Orlicz sequence spaces without local convexity", Proc. Comb. Phil. Soc. 81, (1977), 253-277.

[3] N. J. Kalton, "Banach envelopes of non-locally convex spaces", (To appear).

[4] J. Lindenstrass, L. Tzafriri, Classical Banach spaces: Sequence spaces. Springer-Verlag, Berlin (1977).

[5] J. R. Morrow, "On basic sequences in non-locally convex spaces", Studia Math. 67, (1980), 114-133.

Departamento de Teoria de Funciones

Facultad de Mathematicas

Universidad de Barcelona

Gran Via 585, 08007 BARCELONA,

Spain. 